\title{
EL ESTUDIO DE MEDIO ORIENTE EN LA DISCIPLINA DE RELACIONES INTERNACIONALES EN MÉXICO
}

Marta TAWIL

El inTERÉS POR Medio OrIENTE entre internacionalistas mexicanos ha parecido ir en aumento; es posible documentar el avance en el estudio de los problemas y cuestiones en lo que concierne a las relaciones internacionales de la región desde América Latina. Uno de los ámbitos que pueden ayudar a entender cómo se articula la reflexión sobre el tema son las investigaciones para obtener grado académico, pues allí se reflejan algunas primeras preocupaciones, enfoques, posibilidades y expectativas más o menos informadas. De esa manera se puede colaborar a establecer un examen sólido consistente con los esfuerzos en este campo, los cuales a su vez puedan favorecer la formulación de políticas y el compromiso público de académicos e investigadores.

Se identifican temas dominantes y herramientas metodológicas y teóricas, en esta etapa de la investigación, en tesis de licenciatura y posgrado, que traten sobre temas y países de Medio Oriente y que hayan sido escritas desde 1980 en la disciplina de relaciones internacionales en cuatro universidades mexicanas, ${ }^{1}$ que a su vez han ofrecido un programa de licenciatura en relaciones internacionales desde hace al menos diez años. ${ }^{2}$

${ }^{1}$ Universidad Nacional Autónoma de México (UNAM), Instituto Tecnológico Autónomo de México (ITAm), Universidad de las Américas Puebla (udlap) y El Colegio de México.

${ }^{2}$ Las cuatro universidades ofrecen cursos sobre Medio Oriente y Norte de África. 
Mientras que esta investigación habrá de irse complementando con análisis sobre otros ámbitos de investigación y de políticas, hemos observado hasta el momento una imagen a la vez alentadora y desconcertante. Lo más evidente es que hay una agenda pendiente asociada a problemas en las redes de investigación y documentación, la falta de recursos financieros y las prioridades determinadas por la posición geográfica de México y su relación con Estados Unidos.

\section{LA EVOLUCIÓN DE LAS RELACIONES INTERNACIONALES DE Medio Oriente y la aCAdemia mexicana}

Antes de tratar los temas de investigación y las herramientas metodológicas y teóricas, es necesario indicar que el interés por Medio Oriente en México ha aumentado de forma constante en los últimos quince años. Los factores principales que han contribuido a este crecimiento son de diversa índole. Por lo menos dos son internos: la evolución de la agenda de política exterior de México y el crecimiento exponencial de instituciones que ofrecen programas de licenciatura en relaciones internacionales. En efecto, entre 1980 y 1990 México era uno de los países más cerrados, donde las exportaciones e importaciones constituían menos de 30\% del PIB; en las décadas siguientes, pasó a ser una economía donde este porcentaje subió a más de $65 \%$. Respecto al estudio de las relaciones internacionales, el aumento en las instituciones que ofrecen una licenciatura en esa disciplina, así como en comercio internacional y negocios internacionales, ha sido muy importante. En la actualidad hay 23000 estudiantes de relaciones internacionales en México, más de tres mil profesores en relaciones internacionales y más de 110 instituciones que ofrecen estos programas. Hace sólo veinte años, en 1990, había únicamente tres programas de relaciones internacionales en México (la disciplina nació en 1951).

El surgimiento y aumento del interés por Medio Oriente entre jóvenes internacionalistas en el lapso de unos cuantos años resalta el impacto de cambios regionales y mundiales. Además de los efectos de la globalización y la interdependencia, cuatro momentos 
fueron de particular importancia en México, al igual que en otras partes del mundo, e influyeron en el debate sobre las dinámicas regionales después de la Guerra Fría: el inicio de la Guerra del Golfo y del proceso de paz árabe-israelí en 1991, los acontecimientos del 11 de septiembre de 2001, la invasión y ocupación de Iraq en 2003, y las sublevaciones populares a partir del invierno de 2010-2011. Sobre este último tema, por ejemplo, el número de conferencias, simposios, talleres, cursos y mesas redondas ha sido espectacular. Pero el momento definitorio fue, desde luego, el 11 de septiembre. Después de esos atentados, la gran mayoría de las universidades mexicanas no contaba con profesores necesarios para "explicar" los hechos a sus estudiantes. De forma similar, los funcionarios de la Secretaría de Relaciones Exteriores no parecían tener mucha idea de lo que estaba sucediendo y comenzaron a expresar la necesidad de que profesores y estudiantes de la región les explicaran las consecuencias potenciales de esos acontecimientos para las relaciones entre México y Estados Unidos, con el objetivo de articular mejor las opciones de política exterior. En términos de investigación y pedagogía, el efecto neto de estos elementos (internos e internacionales) sobre los estudios de Medio Oriente en México ha sido, pues, positivo, aunque, como se verá a continuación, no de manera sistemática.

\section{Temas}

En una de las gráficas del anexo es posible apreciar los temas investigados en las tesis escritas por estudiantes de relaciones internacionales. El tema más estudiado es el conflicto árabe-israelí, con treinta tesis escritas al respecto (16.95\%); en segundo lugar, con 21 tesis (11.86\%), viene la relación entre Estados Unidos y los países de Medio Oriente (con un énfasis particular en la política exterior de Estados Unidos); y en tercer lugar 17 tesis estudian las organizaciones internacionales y el derecho internacional (9.60\%). Los países cuyas políticas exteriores hacia la región han sido más estudiadas son, en importancia decreciente, Estados Unidos, Francia y España. Es interesante notar que Irán y Arabia Saudita comienzan 
a llamar más la atención a partir de 2008. Hay una tendencia a estudiar la región no principalmente mediante el análisis de las fuerzas internas, los actores y sus motivos, sino a través de la acción de actores extranjeros.

Existe asimismo interés por analizar las problemáticas palestina, kurda, del Sahara Occidental e iraquí mediante la discusión de normas y principios del derecho internacional. El número de tesis sobre la norma de Responsabilidad de Proteger puede ligarse en parte al activismo tradicional de México en defensa de los principios del derecho internacional. Más aún, el número de estas tesis coincide con el periodo bianual durante el cual México fue miembro no permanente del Consejo de Seguridad de las Naciones Unidas.

No hay tesis sobre la situación de los refugiados palestinos en Líbano ni sobre Jordania. El Magreb o Norte de África en sí no se estudia; recibe atención cuando se examina la política de países europeos hacia esa subregión. Algunos países no habían atraído el interés de estudiantes hasta el inicio de la Primavera Árabe en 2011, como es el caso de Libia y Túnez.

La geopolítica del petróleo y el agua son temas frecuentes en estas tesis (constituyen $6.48 \%$ del número total de tesis analizadas). Algunas de ellas estudian la importancia geopolítica del agua con el enfoque de la teoría de "las nuevas guerras". A pesar del aumento en el interés sobre percepciones, imágenes y medios de comunicación en los últimos tres años, sólo hay una tesis sobre la empresa de medios más grande de la región, Al-Jazeera. Los resultados también muestran que hubo trece tesis donde el islam fue el tema principal; en su mayoría estas tesis analizan la dimensión política del islam en Medio Oriente. Muchas tesis intentan comprender cómo la globalización afecta el resurgimiento del islam, o explicar el islamismo como una reacción a la modernidad.

El interés en la política exterior de México hacia los países del Consejo de Cooperación del Golfo ha aumentado de forma constante desde 2008, lo cual coincide con un renovado interés de México por diversificar sus relaciones económicas, y con la apertura de nuevas embajadas en países árabes del Golfo (desde 2008, México abrió tres nuevas embajadas en Qatar, los Emiratos Árabes Unidos y Kuwait). En cambio, investigaciones sobre Medio 
Oriente no se han inscrito hasta ahora en otros temas prominentes dentro de la disciplina de relaciones internacionales, como la gobernanza global o la cooperación Sur-Sur.

\section{Herramientas metodológicas y teóricas}

Los generalistas de disciplinas suelen criticar a los que hacen estudios de área, regionales, en general, por hacer trabajo a-teórico, esto es, sin utilidad o aplicación universal y sin orientación política que favorezca la agenda estratégica de Washington. Además, se nos dijo que con el fin de la Guerra Fría y los efectos de la interdependencia y la globalización había que mirar el "panorama general" y no lo regional, ya que "todo" se estaba "homogeneizando". ${ }^{3}$

En los últimos quince o veinte años ha habido más sensibilidad en Estados Unidos hacia la elaboración de teoría o su aplicación. Se aplican paradigmas de la economía política, autoritarismos, análisis de política exterior, teoría de las relaciones internacionales. Pero en general se teme o asume que las teorías no tienen el valor explicativo suficiente en el escenario medio-oriental. Esto puede ser un síntoma de un problema más amplio, el de la utilidad limitada de conceptos y teorías estándar elaboradas en Occidente en contextos no-occidentales. Esa es una de las razones por las que muchos estudiantes de Medio Oriente en México, frustrados en sus esfuerzos por hacer que los acontecimientos en esa zona del mundo "quepan" en teorías existentes, se alejen de la teoría y se refugien en el supuesto "excepcionalismo" de esta región; o bien arguyan que ellos presentan los hechos tal y como son, como si hacer eso, sin interpretación conceptual, significara forzosamente que su trabajo es más realista u objetivo. Hay que decir que en esto a veces no ayudan los especialistas del área pertenecientes a otras disciplinas, como la antropología y la historia, que ocupan el terreno de los temas políticos negando a los politólogos e internacionalistas un savoir-faire específico en la materia.

${ }^{3}$ Pinar Bilgin, "What Future for Middle Eastern Studies?", Futures, vol. 38, 2006, p. 575. 
Hay una baja tendencia por hacer teoría por parte de los especialistas internacionalistas en México y América Latina en el estudio de regiones.

Es de hecho una tendencia que caracterizó durante muchos años a los estudios sobre Medio Oriente en Estados Unidos. Rex Brynen ha apuntado como hipótesis al dominio del idioma inglés en los estudios de Medio Oriente y la relación entre el apoyo del gobierno estadounidense y los intereses estratégicos de Estados Unidos. ${ }^{4}$ Sabemos, de hecho, que desde 1945 el desarrollo de los estudios de área reflejó la necesidad de las grandes potencias y de los diseñadores de políticas internacionales de influir en los procesos de cambio y estabilización de Medio Oriente. También puede decirse que mucha de la documentación está diseñada para influir en la formulación de la agenda o el apoyo de los intereses de Estados Unidos. El resultado es que se pone más énfasis en identificar un problema y menos en construir teoría analítica. Por su parte, los que no rechazan explícitamente la importancia de la teoría, acuden al realismo, al neorrealismo y a la lente de los estudios estratégicos, que consideran herramientas más útiles para analizar los procesos en otras partes del mundo fuera de Occidente (nociones de equilibrio de poder, dilema de seguridad, etc.). Los enfoques sistémicos han ofrecido aportes valiosos para el estudio de las relaciones internacionales de Medio Oriente, especialmente porque han demostrado la sensibilidad y vulnerabilidad de la región frente a las variables externas. Sin embargo, por su propia naturaleza, esta perspectiva puede llevar a un cierto desconocimiento de las posiciones y funciones ampliamente distintas que cada país de Medio Oriente ocupa, en el sistema internacional, así como de las variables internas que inciden en su comportamiento externo. El problema es que a veces ese supuesto realismo no siempre se aplica correctamente. Se describen acontecimientos con el lenguaje realista, de una forma poco rigurosa, sin precisión metodológica. ${ }^{5}$

${ }^{4}$ Rex Brynen, "The State of the Art in Middle Eastern Studies: A Research Note on Inquiry and the American Empire”, Arab Studies Quarterly, vol. 8, núm. 4, 1986, pp. 404-419.

${ }^{5}$ Pinar Bilgin, art. cit., p. 581. 
El método más utilizado en las tesis sobre Medio Oriente es el método cualitativo, con 207 tesis $(95.83 \%)$. El método cuantitativo se utiliza únicamente en nueve tesis $(4.17 \%)$. El método comparativo es marginal, ya sea si se utiliza dentro de la región misma o entre el Medio Oriente y otras regiones. ${ }^{6}$ Hay un alto grado de segregación de Medio Oriente (o de la región, o del mundo árabe) en la investigación y en los salones de clase. No obstante, éste es un problema que concierne al conjunto de estudios sobre áreas regionales. Los estudiantes en México apenas entran a debates interregionales y genuinamente comparativos. Tres áreas temáticas han sido particularmente atractivas -y prometedoras- en los últimos tres años: 1) poderes medios y poder regional (México y Turquía, Venezuela e Irán), 2) política exterior de países en transición democrática (América Latina en la década de 1980 y países árabes desde 2011) y 3) desde una perspectiva más sociológica, el legado del colonialismo en el Medio Oriente y el Norte de África y en América Latina.

En el plano de la teoría de relaciones internacionales, pareciera que se asume que una única teoría o un solo nivel de análisis ofrecen un mecanismo satisfactorio para explorar ya la dinámica cambiante de la política interregional, ya las políticas internacionales de la región o ya para explicar por qué altos niveles de cooperación coexisten, en ocasiones, con altos niveles de competencia y conflicto.

El análisis a nivel sistémico es, de lejos, la herramienta privilegiada: realismo clásico, neorrealismo y realismo estructural (poder

${ }^{6}$ Muchos países de Medio Oriente comparten ciertos intereses y experiencias con México, América Central y el Cono Sur: el alto valor otorgado a principios como el derecho a la auto-determinación y la soberanía de los Estados; problemas similares relaciones al proceso de liberalización económica y política; la regionalización informal de flujos laborales y redes sociales complejas; el factor religioso como un marco para grupos sociales y movimientos políticos; la regionalización del discurso institucional y de las prácticas en términos de seguridad, promovidas tanto por las políticas de los grandes poderes como por el fenómeno de la globalización; el problema de la violencia política y social, el comercio de armas ligeras y la carrera armamentista; la degradación ambiental y la escasez de recursos como el agua, etc. Desafortunadamente, no se ha reconocido aún que estos y otros asuntos nos unen en una dinámica de interdependencia inescapable. 
e interés nacional, seguridad, alianzas, hegemonía) vienen primero; luego se recurre al paradigma constructivista (identidad, cultura); y, en tercer lugar, los estudiantes se inclinan por el liberalismo (teoría de la paz democrática, instituciones, cooperación). Hay una obsesión con la geopolítica, en el sentido de que Medio Oriente interesa sólo por su impacto en la seguridad internacional.

Por lo que respecta a las explicaciones del segundo nivel, o nivel estatal, son todavía escasas. La seguridad del régimen político y un rango de consideraciones, incluidas las relaciones entre Estado y sociedad, no se perciben como cruciales para comprender cómo los países de Medio Oriente se posicionan en el ámbito internacional. Sólo 3.39\% de las tesis estudian el sistema político israelí como una manera de comprender la política exterior de Tel Aviv y sus estrategias de negociación con los palestinos; la religión nunca se toma como variable intermedia o independiente. Las explicaciones sobre el conflicto árabe/palestino-israelí son en su mayoría sistémicas, y no buscan respuestas dentro de Palestina o Israel.

Sólo en fechas muy recientes ha surgido mayor conciencia sobre el hecho de que el análisis de tercer nivel, o sistémico, es inadecuado, y que para entender el comportamiento en el sistema internacional de los países de Medio Oriente se requiere un marco teórico flexible e incluyente. En consecuencia, es posible encontrar ahora algunas tesis que combinan enfoques realistas y estructuralistas, con elementos de constructivismo. Los estudios siguen, sin embargo, marginando a la sociología de las relaciones internacionales, la cual busca explicar los hechos internacionales como hechos sociales o que integran preocupaciones sociales.

El predominio de explicaciones sistémicas no es sorprendente ni exclusivo de México. Medio Oriente tiene una larga historia de interferencia externa y dependencia, lo que explica el atractivo del realismo y del estructuralismo. Más aún, las tesis de licenciatura, que sobrepasan las tesis de maestría y doctorado en la muestra, no requieren un alto grado de especialización.

Cuando se utiliza la teoría, su lectura es a la vez empírica y normativa. Esta intersección es útil. Todas las teorías de relaciones internacionales y política global tienen importantes dimensiones 
empíricas y normativas, y su profunda interconexión es inevitable. Muchas tesis revelan que la motivación de los estudiantes es la pregunta práctica de cómo se debería actuar. Todas las teorías contienen elementos normativos significativos por medio de las preguntas que se hacen, los conceptos que usan, los factores que excluyen o consideran constantes y los valores que buscan promover.

En términos generales, cualesquiera que sean los acontecimientos en la región -violencia o revueltas antiautoritarias-, las tesis tienden, en forma variable, a separarlos de relaciones de poder locales, regionales e internacionales, para favorecer variables más culturalistas, que son, en la mayor parte de los casos, esencialistas, reduccionistas y unidimensionales. Además, lo que supuestamente no sucede en la región también se atribuye a los mismos tropos esencialistas -esto es, democracia, secularismo, paz y desarrollo- sin importar cómo estos términos se definen o si son deseables así definidos. Esta tendencia se encuentra sobre todo en las tesis que estudian mujeres (políticas de género), islam y terrorismo. Los escritos "orientalistas" no tienen, en consecuencia, el monopolio de los problemas. La problemática del Sahara Occidental o Palestina son los temas principales analizados con la lente de la conspiración. En ocasiones, el discurso anti-hegemónico es igual de problemático que el discurso hegemónico. En consecuencia, al cuestionar las acciones de los países occidentales, muchas tesis incurren en las mismas explicaciones existencialistas que ven en el islam la respuesta a todo.

IV. El PAPEL DE INVESTIGADORES Y ACADÉMICOS EN LA aRTiculación de la POLÍtica EXTERIOR de MÉXico

¿Cómo estos hallazgos se relacionan con, o se traducen en, la capacidad -potencial o real- de los internacionalistas mexicanos con un interés especial en Medio Oriente de desempeñar un papel en estimular la investigación y las discusiones públicas, y en contribuir a la formulación de la política pública?

Para esbozar una primera respuesta a esta pregunta, se puede revisar el número de instituciones en México que publican libros 
o revistas académicas sobre Medio Oriente. Descubrimos que ese número es limitado. El Colegio de México es la institución que más publica sobre el tema en el país desde 1980 (alrededor de treinta libros, una revista académica especializada y una revista sobre relaciones internacionales). Además, es un hecho que el número de publicaciones desde 1980 sobre las relaciones internacionales de Medio Oriente o sobre Medio Oriente en las relaciones internacionales ha aumentado notoriamente. ${ }^{7}$ Esta evolución no es desdenable. Con todo, los investigadores mexicanos con un título en relaciones internacionales, y con conocimiento de Medio Oriente, no se interesan en contribuir al desarrollo de teorías, por ejemplo sobre alianzas, regionalismo, guerra, cooperación, etcétera.

Esto debiera ser una preocupación central no sólo para el diminuto e insular mundo de los estudios sobre Medio Oriente en México, sino también para todos los académicos preocupados por la negligencia, y en ocasiones la politización, del apoyo gubernamental para la investigación académica sobre el mundo árabe. Esto conduce a la segunda y última pregunta mencionada arriba. Muchos funcionarios en la Dirección General para África y Medio Oriente de la Secretaría de Relaciones Exteriores son conscientes de que saber más sobre Medio Oriente es algo bueno. Con todo, los estudios sobre Medio Oriente y el entrenamiento en idiomas no han sido apoyados de forma consistente ni adecuada por las instituciones mexicanas ni por el gobierno, y parecen quedar reducidos a la anécdota y a la superficialidad.

En 2012, una joven estudiante mexicana de conocimiento de la autora solicitó ingresar a una maestría en la Universidad Americana

${ }^{7}$ De 1980 a 1990 se publicaron cuatro libros (0.4 libros por año); de 1990 a 2000, siete libros (0.7 libros por año); de 2000 a 2010 se publicaron 19 libros (1.9 libros por año); y, desde 2011, se han publicado siete libros (1.75 libros por año). Pocas revistas académicas en México han publicado artículos sobre Medio Oriente. Las principales son las siguientes: Estudios de Asia y África, Foro Internacional, Revista de Historia Internacional, Revista Mexicana de Política Exterior, Revista de Relaciones Internacionales, Tendencias, Foreign Affairs en español y Nexos. Las revistas académicas Estudios de Asia y África y Foro Internacional, publicadas por El Colegio de México, han publicado el número más grande de artículos sobre la región (12 y 17 , respectivamente). 
en El Cairo. Una vez admitida en el programa, pidió una beca ante una institución federal mexicana, Conacyt. La respuesta que recibió fue que su tema y su estancia en El Cairo eran "inconsecuentes en términos del interés práctico para México”. Hay otra anécdota sobre estudiantes del programa de licenciatura en Relaciones Internacionales de una prestigiosa institución, quienes buscaban opciones de intercambio académico en el extranjero. A los estudiantes que querían ir a El Cairo o a Beirut se dijo que esos lugares eran peligrosos (los que querían pasar su séptimo semestre en Rusia afrontaron una falta de apoyo similar; la administración argumentó que Rusia era una país autoritario, y por ende, peligroso). Si por una parte es comprensible la preocupación sobre el bienestar de los investigadores en ciernes, y debería planificarse, hasta allí avanzó el plan de apoyo a esas investigaciones.

De esa manera, parece asumirse que sólo especialistas en Estados Unidos o Europa pueden elaborar conocimiento sobre Medio Oriente, y que trabajar desde América Latina nos confina a estudiar esa región sin precisión, bajo lentes ideológicas o a la distancia, haciéndonos incapaces de cualquier investigación académica rigurosa. Algo similar ocurre en los circuitos del gobierno. Funcionarios, sobre todo de la Secretaría de Relaciones Exteriores, llaman de manera frecuente a especialistas en Medio Oriente (académicos, pero también a estudiantes) de distintas disciplinas para que compartan sus conocimientos y provean consejos de política pública sobre coyunturas particulares o momentos de crisis (cualquier crisis excepto cuando se trata de Israel). Más allá de eso, no hay un seguimiento de los acontecimientos. La situación preocupa mientras permanece en las páginas de los periódicos. Esto es verdad a pesar de que México se interesa más en Medio Oriente (y le atraen las perspectivas económicas de relaciones más profundas y extensas con los países del Golfo Árabe), y con todo y haber creado una Oficina de Planeación y Estrategia dentro de la Secretaría de Relaciones Exteriores.

Una paradoja caracteriza al campo de los estudios de Medio Oriente en el mundo occidental, y muy en particular en el continente americano, aunque también ocurre en Europa. Por un lado, especialmente desde el 11 de septiembre existe un amplio 
reconocimiento de que se necesitan urgentemente un conocimiento experto y una comprensión más profunda de Medio Oriente y del mundo islámico, por el sinnúmero de retos para la seguridad internacional, por los problemas enormes de percepción, desconfianza y hostilidad. Ya sea en la arena de la democratización, los derechos humanos, el extremismo religioso y el terrorismo internacional, la proliferación nuclear, en lidiar con las consecuencias de la invasión a Iraq, contribuir a las negociaciones entre palestinos e israelíes o en enfrentar a la política iraní, Medio Oriente continúa en el centro de las preocupaciones de la política exterior estadounidense. En el plano de la opinión pública, en Norteamérica y otras partes del globo, se observa un resurgimiento del interés por Medio Oriente, en particular desde los trágicos acontecimientos del 11 de septiembre; ese interés se refleja en la publicación de numerosas obras (académicas y de divulgación), mayor cobertura de medios de comunicación y el aumento en la popularidad de cursos y seminarios sobre las lenguas, culturas y la política de Medio Oriente en los campus de Estados Unidos, Canadá, países europeos y también en México.

Por otro lado, sin embargo, el campo de los estudios de Medio Oriente y muchos de sus profesionistas en Estados Unidos y Reino Unido paralelamente han enfrentado una ola de críticas, intimidaciones y acusaciones de una supuesta parcialidad ideológica y distorsión de la verdad, de ser "antiamericanos", "pro-islámicos" y "pro-palestinos" en sus cursos y escritos, y de ser antisemitas cuando elaboran o expresan críticas a las políticas de Israel. ${ }^{8}$ Figuras religiosas evangélicas en Estados Unidos que califican al profeta Mohamed como un terrorista o al islam como una religión intrínsecamente violenta lamentablemente se han vuelto comunes. Son conocidas las denuncias que estos expertos y sus universidades en Estados Unidos y el Reino Unido han hecho de la caza de brujas que han padecido, apuntando a la amenaza que ésta representa a los derechos de la libre expresión e investigación, así como a la integridad personal y de las instituciones. La razón de

${ }^{8}$ Mervat Hatem, "MESA President, Letter from the President Mervat Hatem" MESA Newsletter, vol. 30 núm. 1, febrero de 2008. 
esa intolerancia ha sido posible por el clima político que prevalece en Estados Unidos desde que éste declaró su "guerra global contra el terrorismo".

En México no se ha registrado ese problema a esas dimensiones ni como acción concertada desde las esferas del Estado, pero sí existe la censura y la autocensura específicamente cuando de criticar a Israel se trata (se acusa automáticamente de antisemitismo o de parcialidad irreflexiva pro-palestina), o cuando se habla de la dimensión primordialmente nacionalista de grupos como Hamás o Hezbolá, o cuando se busca dar a conocer la complejidad de la situación de Irán o exigir datos robustos que confirmen los vínculos entre Hezbolá y los Zetas (y que se interpreta en algunos círculos gubernamentales, institucionales e incluso académicos, como una postura a favor de las armas nucleares y del antisemitismo). O sea que aquí también se politiza el estudio de ciertos temas. No es fácil que los estudios eviten considerar las implicaciones políticas y el contexto político de la investigación. El rasgo político inescapable de toda investigación académica es particularmente obvia en Medio Oriente, donde la historia siempre ha sido un campo de batalla de perspectivas ideológicas.

Por último, se nota que México sigue teniendo una política que disocia los asuntos económicos de los políticos y de las dinámicas de las subregiones de Medio Oriente. Esto resulta en una falta de estrategia. La tendencia general que ha prevalecido hacia Medio Oriente puede denominarse "neoliberal", que combina neoliberalismo en asuntos económicos con una tendencia pro-estadounidense en política exterior, en particular en temas de "alta política”. Las redes alrededor de la presidencia priorizan los asuntos económicos con América del Norte. Sólo recientemente, bajo la administración del presidente Peña Nieto, México ha dado mayor atención a sus vecinos del sur, y ha aumentado también sus relaciones con los países del Pacífico asiático. No obstante, la política económica es la principal guía que orienta a la acción diplomática. Más aún, los miembros clave del equipo de política exterior ven la globalización no sólo en términos económicos neoliberales, sino mediante la lente de la agenda de seguridad nacional de México y Estados Unidos. Podría argumentarse que esta tendencia está unida a las conse- 
cuencias de la decisión de México en los años noventa de integrarse completamente a la región norteamericana. Las consecuencias de este posicionamiento estructural han sido duraderas y son difíciles de cambiar a corto y mediano plazo. Esto no quiere decir que la política exterior mexicana hacia Medio Oriente sea ideológica. La persistencia de la tendencia neoliberal da incluso espacio a posturas pragmáticas que se consideran congruentes y benéficas para proyectos internos. El gobierno de Enrique Peña Nieto ha aprobado una serie de reformas de los marcos legales en los temas de energía, trabajo y comunicaciones, las cuales conducen a su (mayor) liberalización. Dichos proyectos y reformas se dirigen a garantizar la estabilidad macroeconómica y expandir el crecimiento, lo que a su vez se percibe como una manera de sostener el regreso de México a la escena internacional como "potencia media".

En consecuencia, no se trata sólo de la academia mexicana; es también un asunto de política exterior y de la relación de México con Estados Unidos. Los datos recabados hasta este momento muestran que los problemas en ambos ámbitos son frenos para el desarrollo cumulativo de los estudios sobre Medio Oriente en México, así como el potencial de estudiantes e investigadores en el campo de las relaciones internacionales de contribuir a acercar México a Medio Oriente.

Traducción de María Fernanda López Portillo Alcocer 
Anexo

\begin{tabular}{|c|c|c|}
\hline Tema & Absoluto & Porcentaje \\
\hline Conflicto Árabe-Israelí & 31 & 14.35 \\
\hline Estados Unidos-Medio Oriente & 25 & 11.57 \\
\hline Organizaciones internacionales y derecho internacional & 18 & 8.33 \\
\hline Islam & 15 & 6.94 \\
\hline Magreb & 14 & 6.48 \\
\hline Irán & 17 & 7.87 \\
\hline Iraq & 12 & 5.56 \\
\hline Guerra del Golfo/Golfo Árabe-Persa & 12 & 5.56 \\
\hline Recursos naturales & 14 & 6.48 \\
\hline Medio Oriente y América Latina & 10 & 4.63 \\
\hline Israel & 6 & 2.78 \\
\hline Medio Oriente-Unión Europea & 10 & 4.63 \\
\hline Terrorismo & 9 & 4.17 \\
\hline Primavera Árabe & 4 & 1.85 \\
\hline Hamás & 6 & 2.78 \\
\hline Turquía & 4 & 1.85 \\
\hline Líbano & 4 & 1.85 \\
\hline Crisis siria & 2 & 0.93 \\
\hline Zionismo & 1 & 0.46 \\
\hline Medios & 1 & 0.46 \\
\hline Economía & 1 & 0.46 \\
\hline Total & 216 & 100 \\
\hline
\end{tabular}




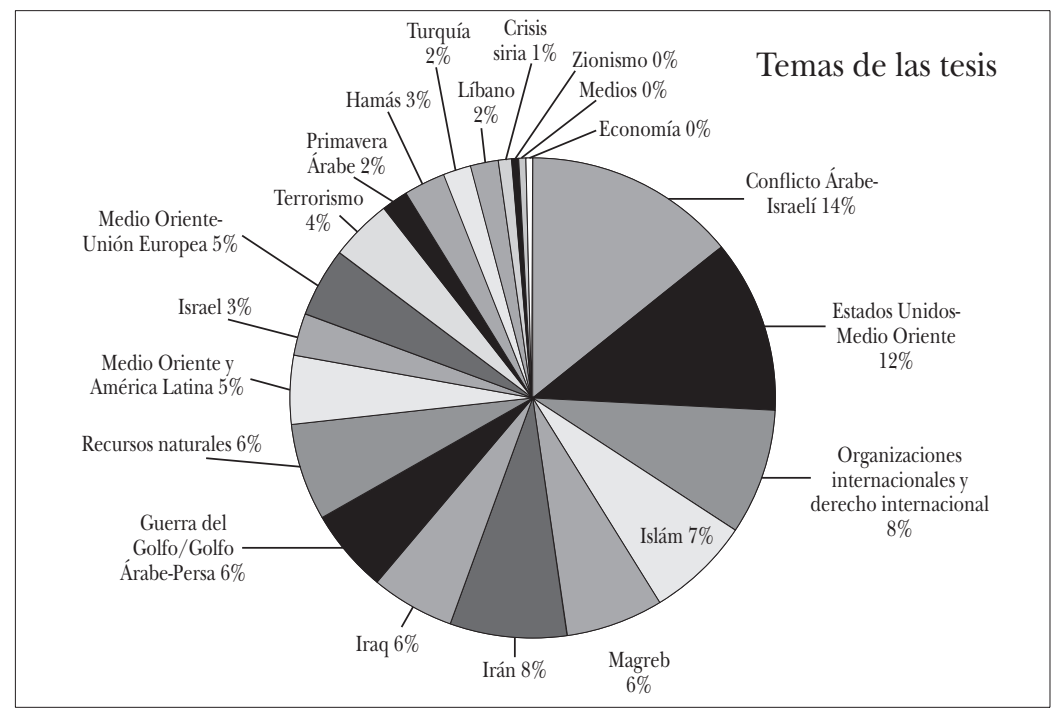




\begin{tabular}{rcc}
\hline Método & Absoluto & Porcentaje \\
\hline Cualitativo & 207 & 96 \\
Cuantitativo & 9 & 4 \\
Total & 216 & 100 \\
\hline
\end{tabular}

Método

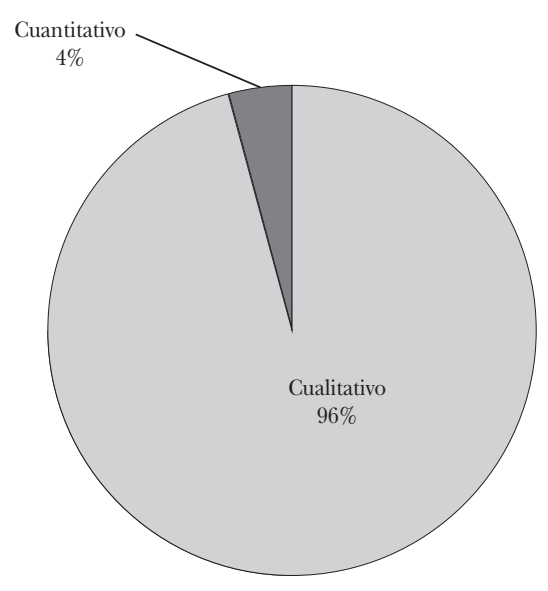




\begin{tabular}{lcc}
\hline \multicolumn{1}{c}{ Uso teórico } & Absoluto & Porcentaje \\
\hline Sin teoría específica & 78 & 36.11 \\
Herramientas teóricas especificadas & 138 & 63.89 \\
Total & 216 & 100 \\
\hline
\end{tabular}

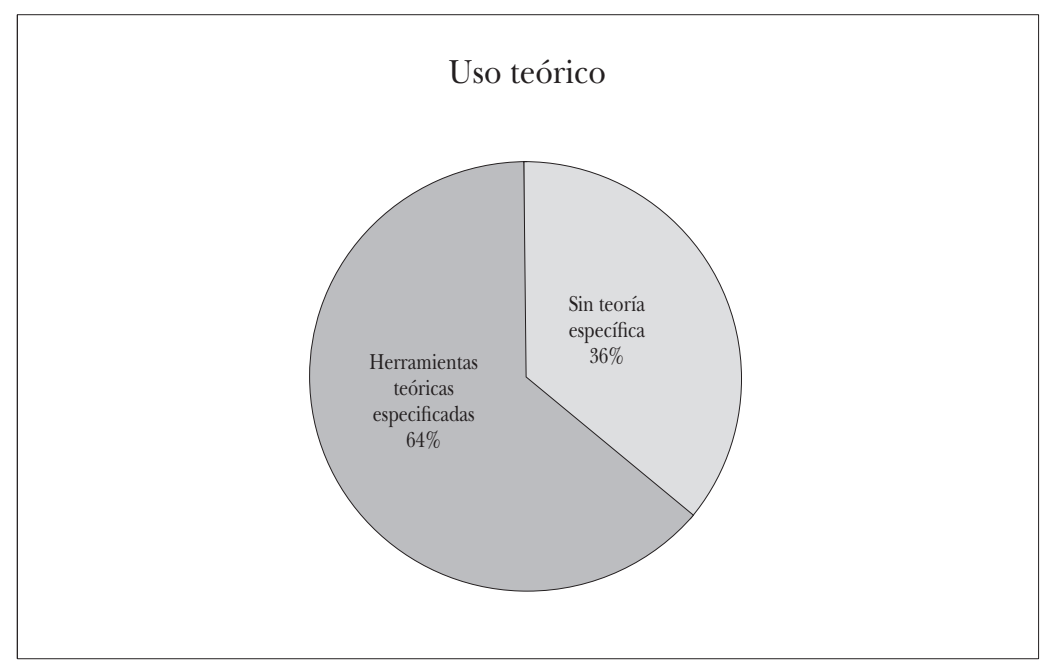




\begin{tabular}{lcc}
\hline \multicolumn{1}{c}{ Teoría } & Absoluto & Porcentaje \\
\hline Teorías sistémicas & 6 & 2.78 \\
Constructivismo & 9 & 4.17 \\
Marxismo y neomarxismo & 14 & 6.48 \\
Sociología histórica / Teorías sociológicas & 16 & 7.41 \\
Liberalismo & 30 & 13.89 \\
Realismo y neorealismo & 64 & 29.63 \\
Sin teoría especificada & 77 & 35.65 \\
\multicolumn{1}{c}{ Total } & 216 & 100 \\
\hline
\end{tabular}

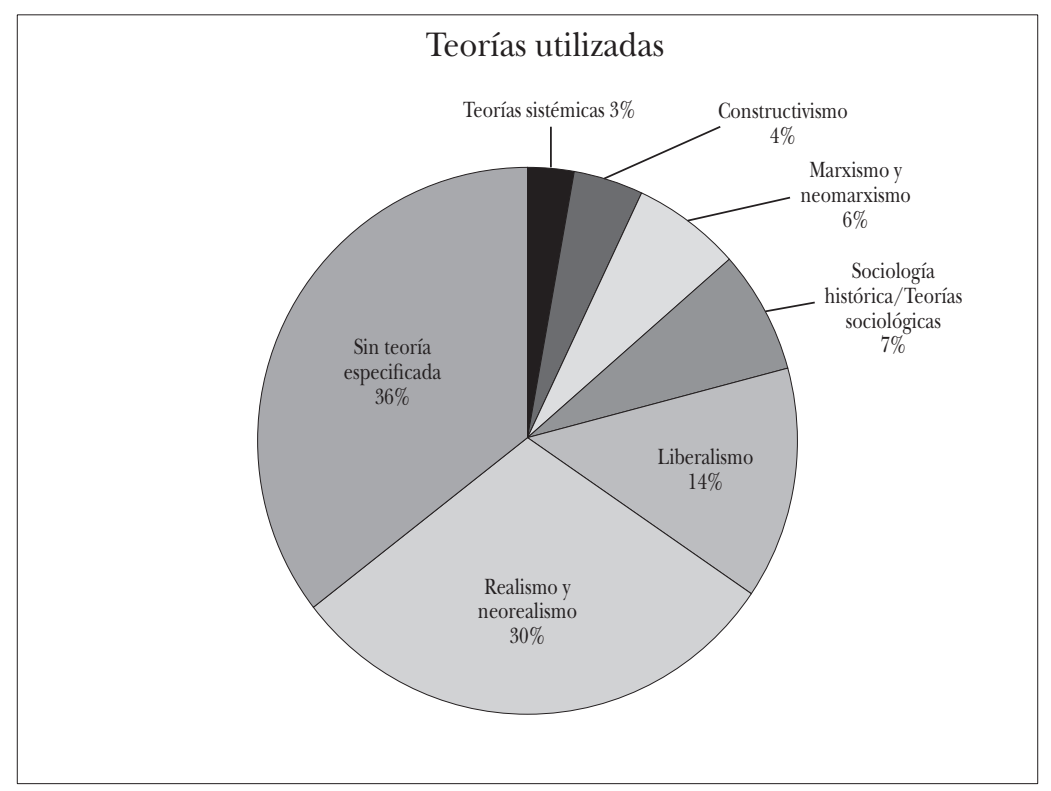

